\title{
Improved Adaptive Optics Operations at the LBT
}

\author{
Gregory E. Taylor ${ }^{a}$, Douglas, L. Miller ${ }^{a}$ Julian C. Christou ${ }^{a}$, Xianyu Zhang ${ }^{a}$, Guido Brusa \\ Zappellini $^{a}$, Pedro Escarate Monetta ${ }^{a}$, Michael Lefebvre ${ }^{a}$, Marco Xompero ${ }^{b}$, Alfio Puglisi ${ }^{b}$, \\ Luca Fini ${ }^{b}$, Enrico Pinna ${ }^{b}$, Simone Esposito ${ }^{b}$. \\ ${ }^{a}$ LBTO, Tucson, Arizona, USA; \\ ${ }^{b}$ INAF, Padua, Italy.
}

\begin{abstract}
The goal for the adaptive optics systems at the Large Binocular Telescope Observatory (LBTO) is for them to operate fully automatically and to be able to be run by the observers or the telescope operator, without the need for an AO Scientist, except for on-call support. We will discuss the improvements which have been made to further this goal, and their application.
\end{abstract}

Keywords: Adaptive Optics, Large Binocular Telescope Observatory

\section{INTRODUCTION}

The First Light AO System (FLAO) ${ }^{1}$ operations at the Large Binocular Telescope (LBT) ${ }^{2,3}$ are becoming routine and several recent improvements have increased the on-sky efficiency of AO observations. A new state machine has been implemented in the AO Supervisor software. This state machine will catch errors during loop closure and during operation. This allows user intervention and fault recovery, during nighttime operations. Web based troubleshooting and recovery guides have been created to aid the Observing Service Associate, Science Support Observers and AO staff. New routines have been developed to process the AO Supervisor software logs and report operations information, including troubleshooting guidelines to the user. Finally, new routines have been created to allow for a rapid readiness checkout of the $\mathrm{AO}$ system at the beginning of the night.

\section{UPGRADED AO: UAO}

Over the past two years, the FLAO team has been working on improving the the software and low-level firmware that controls the FLAO system. These software and firmware improvements constitute what we call Upgraded AO (UAO). They will be discussed in the following subsections.

\subsection{Improved firmware in the Adaptive Secondary}

New firmware has been implemented in the Adaptive Secondaries (AdSecs) ${ }^{4}$ at the LBT. Previously, if an actuator position measurement within the AdSec was outside of defined limits, the AO loop was opened and the shell rested ( $\mathrm{see}^{5}$ for a description of the shell resting). These measurements are made with a capacitive sensor that measures the separation of the AdSec shell from the reference body at a rate of $70 \mathrm{KHz} .{ }^{4}$ With the new firmware, the nearby actuator positions are checked for verification. Only if all four actuators on a single Digital Signal Processor (DSP) channel register measurements which are out of bounds, will the shell be rested. This allows the shell to be kept safe while rejecting spurious measurements which could be due to any number of issues with the electronics. Our AdSecs are prototypes nearing 10 years of age. ${ }^{5}$ Hence there are spurious measurements at times.

Further author information: (Send correspondence to G.E.T.)

G.E.T.: E-mail: gtaylor@lbto.org, Telephone: 15206266732 


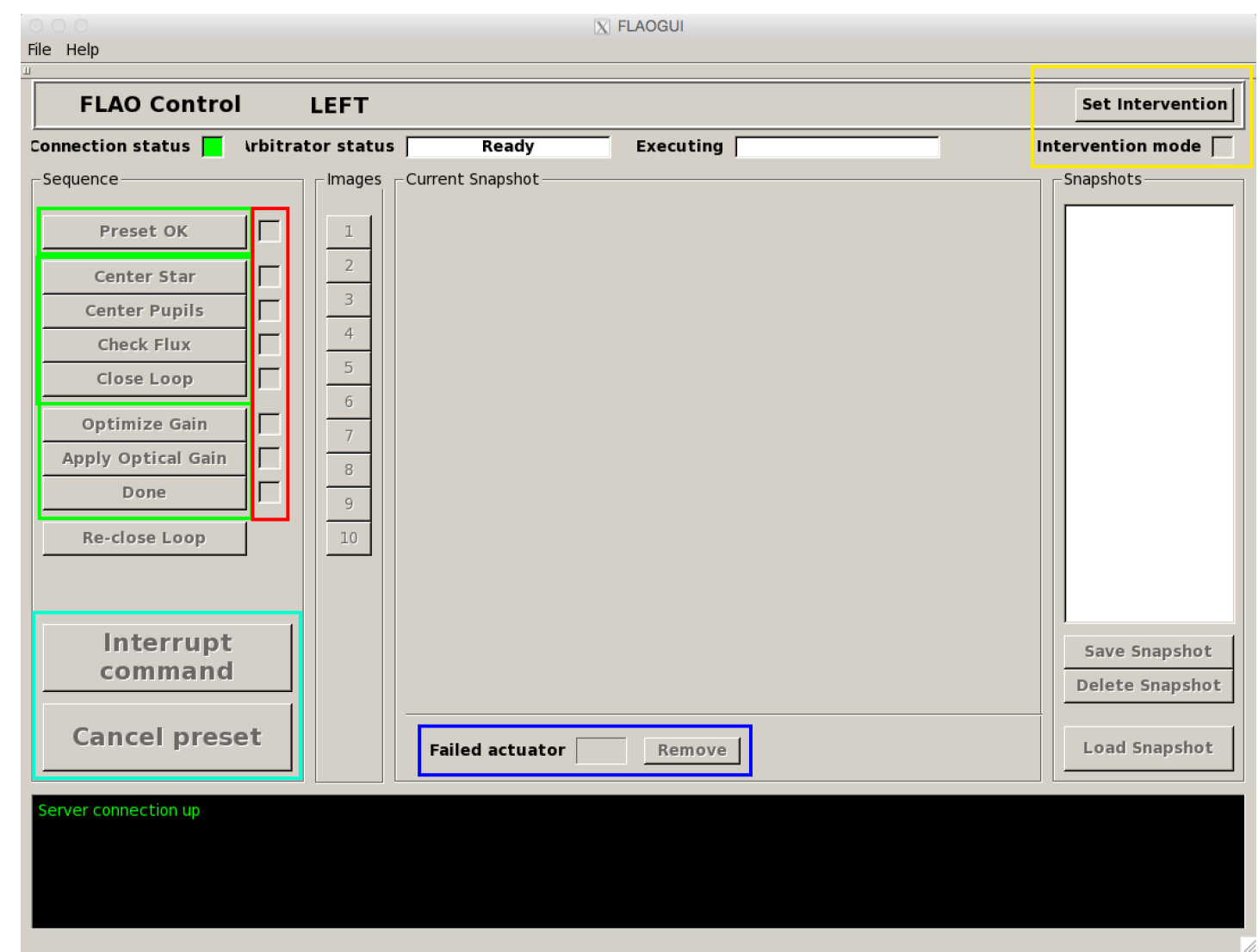

Figure 1. The FLAO GUI. This is a prototype GUI which provides more advanced functionality than previously available. In the "Intervention Mode", the user can verify that each step is successfully completed, re-do failed steps, after adjusting one or more operational parameters, or do a step completely manually and skip the step on the GUI.

\subsection{Improved AO Closed Loop State machine}

Previously, closing the AO Loop was all or nothing. If any one step failed, all steps had to be repeated. An intervention mode allows the state machine pause the process of closing the loop when a step fails. The OSA can then rectify any problem and resume at that step. This shortens the time required to re-close the loop from four or five minutes to as little as 20 seconds.

These improvements are implemented in a GUI which we call the FLAO GUI. This FLAO GUI is a prototype which has much of the functionality that we would like to have in the future. We would eventually like to implement more advanced functions, such as the ability to automatically recover from specific types of failures. A typical failure is the loop opening due to a rapid, but short-lived change in seeing. This type of failure now requires two steps to fix, taking up to a minute to recover. Even so, the FLAO GUI, and the functionality that it allows, permits the operator to recover from many errors without having to send a new "preset" command to the telescope, which takes as long as 5 minutes to complete. Additionally, "Intervention Mode" allows the user to do a step completely manually, skipping the automated process altogether. This happens most often when attempting to push the limits of the FLAO system's capabilities, not during routine operation.

\section{ROUTINE OPERATIONS WITH FLAO}

\subsection{Web Based Troubleshooting Guide}

As AO operations become routine, the Observing Support Astronomer (OSA) will be called upon to run the FLAO system for normal operations. To that end, a web based troubleshooting guide has been developed which will allow the OSA to find and rectify most routine problems. An AO scientist would be on-call for advanced 


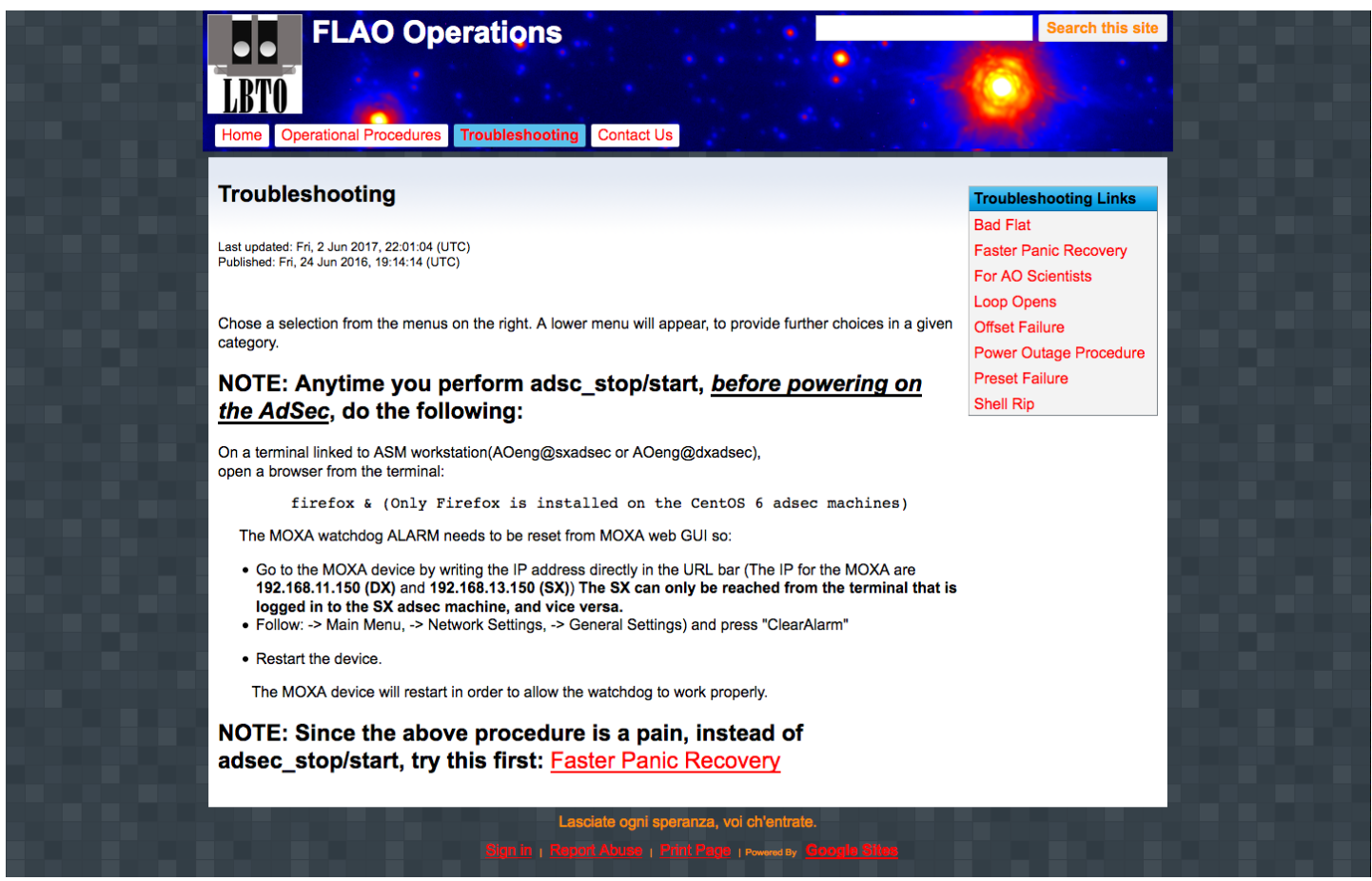

Figure 2. The splash-page of the troubleshooting section of the web-based guide.

troubleshooting. Routine problems generally include: the AO loop opening for one of several reasons, the failure of an offset, the failure of a telescope preset, and any number of more general software issues. These issues require the restarting of a software process, a five second procedure. The more advanced sections, for AO Scientists, detail the removal of faulty actuators from the control loop, calibration procedures, etc. The web-based guide will be adapted to the needs of the OSA as time goes by.

\subsection{Start-up and Checkout}

A new, easy to read GUI has been developed that will allow the Telescope Operator to determine the status and readiness of the various $\mathrm{AO}$ subsystems at a glance. It also allows the user to start and stop the software processes on each subsystem while taking care of potential conflicts, for example, processes running under the ARGOS user account when FLAO is desired.

\subsection{Optical Gain (GOPT)}

A further improvement has been the addition of a process to monitor what we term the Optical Gain (GOPT) of the AO system. Due to the fact that Pyramid Wavefront Sensor (PWFS) is not linear with respect to the strength of wavefront aberrations, ${ }^{6,7}$ a process has been developed in which a small disturbance in $\mathrm{Z} 4$, astigmatism, of a known frequency and amplitude is injected into the system. This disturbance is too small to degrade image quality and fast enough that it isn't corrected by the AO system. The system knows how strong this disturbance actually is, so it compares the expected value with the measured one and adjusts the gains of the system accordingly. As the seeing changes, this measured ratio will also change. Thus, the GOPT process adjusts the gains of the $\mathrm{AO}$ system according to the seeing. This makes the system very robust. Also, any measured Non-Common Path Aberration (NCPA) corrections need to be applied at the correct amplitude, and GOPT ensures that this is the case (See Section 4.1 for a definition of NCPA). This is because in monitoring the ratio between the applied disturbance and the measurement of this disturbance, the correct, calibrated amplitude of NCPA correction will be applied. 

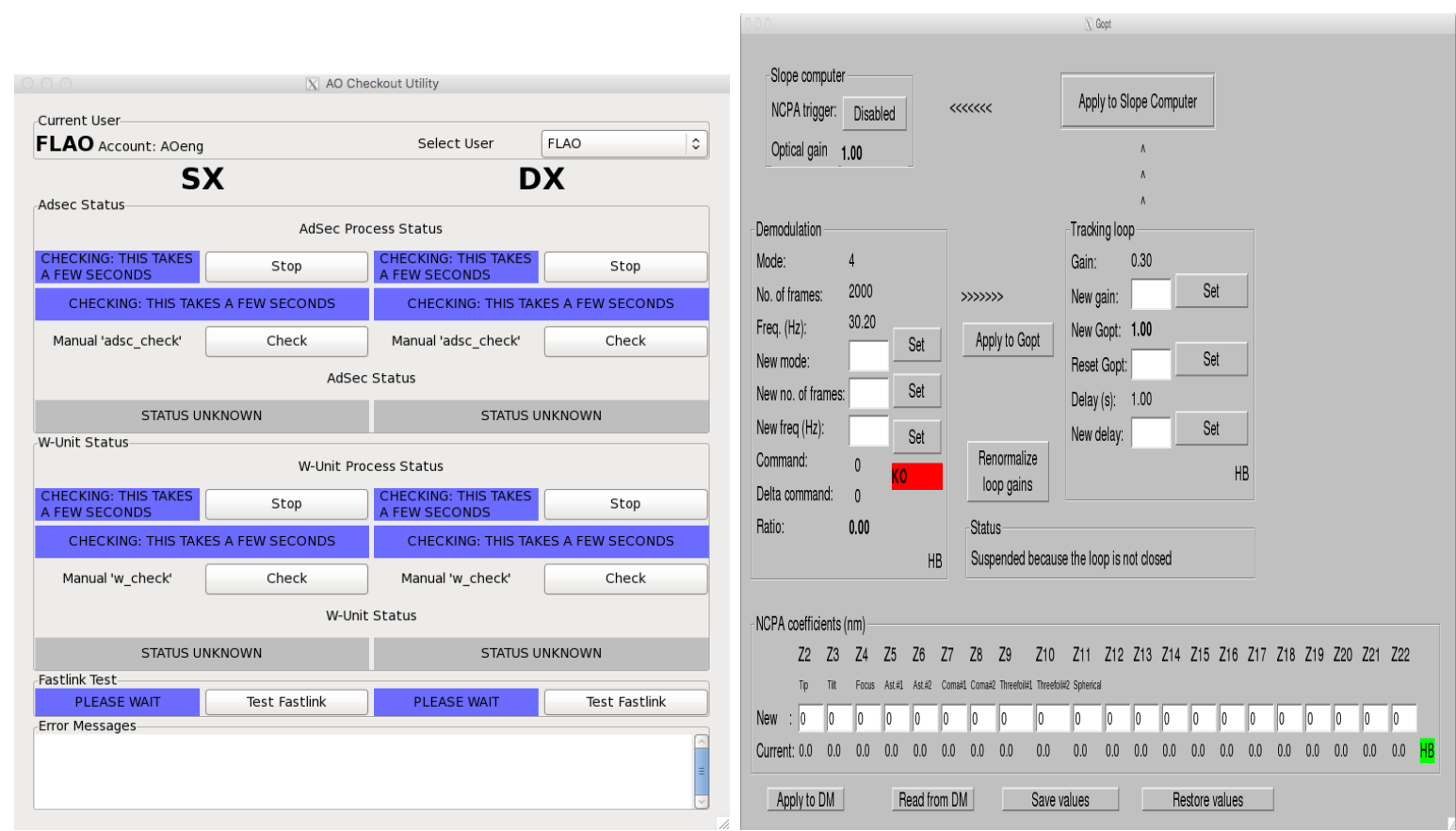

Figure 3. Left: the AO start-up and monitoring utility. This lets the operator see, at a glance, the status of each subsystem, as well as letting him or her start and stop various parts of the software. Right: The GOPT GUI. This GUI allows the user to monitor the status of the "Optical Gain", the way in which the system adapts to changes in seeing, as well as the status of NCPA corrections (See Section 4.1.)

\section{IMPROVING OPTICAL PERFORMANCE}

\subsection{Non-Common Path Aberration Correction}

The FLAO system feeds a pair of near infrared imaging spectrometers named LUCIFER (LUCI). ${ }^{8}$ Since the LUCI imagers contain several optical elements which the FLAO PWFS cannot see, any aberrations created by these optics will not be corrected by the FLAO system. These are so-called Non-Common Path Aberrations (NCPAs)(See [9], for example).

NCPAs are measured by first estimating rough values of Zernike modal coefficients using a software tool and optimizing by-eye using real-time LUCI images. Eight modes are measured, focus through Spherical Aberration (Z11, in the Noll numbering scheme). ${ }^{10}$ The goal is to obtain as high a Strehl Ratio ${ }^{11}$ as possible, with a complete, first diffraction ring, which in our experience is the fastest way to measure NCPAs. The NCPA in each LUCI depends upon its rotator angle, the angle between the imager and the telescope, so NCPA measurements are made at a 30 degree interval of rotator angle and a function is fit to each measured Zernike coefficient (see Figure 4.1). This allows the correct NCPA correction amplitude to be applied for any rotator angle. These fitted values are entered into a Look-Up-Table (LUT), that the FLAO system uses to apply NCPA corrections for each LUCI instrument. We have found no significant NCPA dependence on telescope elevation.

As of now, the NCPA correction routine is started manually, but we are working on including some simple scripts to automatically start NCPA correction. The scripts are now functional, but they need to be installed into the complex FLAO software system.

Any NCPA corrections need to be applied at the correct amplitude and the GOPT process ensures that this is the case (See Section 3.3). This is because in monitoring the ratio between the applied disturbance, described in Section 3.3, and the measurement of this disturbance, the correct, calibrated amplitude of NCPA correction will be applied. 


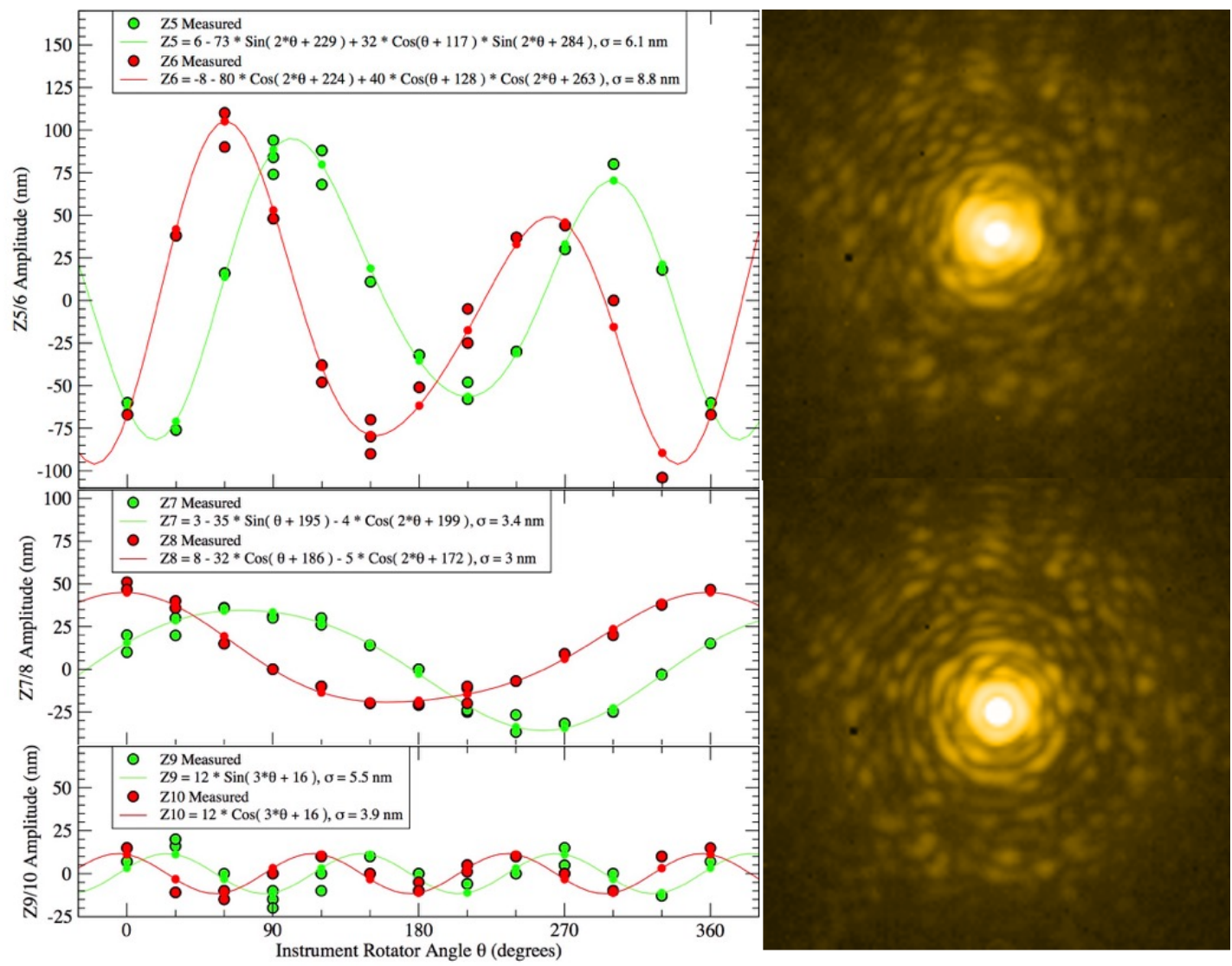

Figure 4. Left Top, Middle, Bottom: Functional fits of Z5 - Z10, for the Right Hand (DX) LUCI camera. Right Top: A star image taken with the DX LUCI (LUCI2) at a rotator angle with a small amount of NCPA present. Right Bottom: An image of the same star with NCPA correction applied.

\subsection{Image Motion Correction}

In as complex an optical system as FLAO/LUCI, it is possible that there can be slight misalignment of optical elements. This occurs in spite of the most careful, in-lab optical alignments. In the case of the Left-Hand (SX) LUCI, LUCI1, there is enough of a misalignment that the image on the focal plane will appear to wobble by almost an arcsecond, as the instrument rotator rotates by 360 degrees. This instrument rotation is needed due to image rotation caused by the Altitude-Azimuth telescope mounting as it tracks an object across the sky. ${ }^{3}$ For normal imaging, exposures are so short that little image motion occurs during any given exposure. However, during spectroscopy, we have noticed that correction of this motion is essential for keeping the source on a narrow, AO-fed spectroscopic slit.

Part of a PWFS is a Tip-Tilt (TT) mirror, which modulates the image of a star around the tip of a glass pyramid. ${ }^{6,7}$ An offset to this modulation can be applied, to correct for the motion of the image on LUCI. This is because there are mechanical stages which will move to re-center the moving image on the tip of the pyramid, once the offset is performed. A TT voltage offset Look-up Table (LUT) has been implemented in the PWFS, in order to correct for residual image motion in the LUCI image plane when the instrument rotator moves. This TT LUT has reduced residual image motion from nearly an arcsecond to a few tens of milliarcseconds (mas) RMS, on LUCI1. LUCI2 has a much smaller image motion, which we also correct via a LUT. We think that any remaining image motion is due to mechanical hysteresis. Further work is on-going to make this as TT LUT application as robust as possible. Even so, it is a vast improvement over what we were able to do previously. 

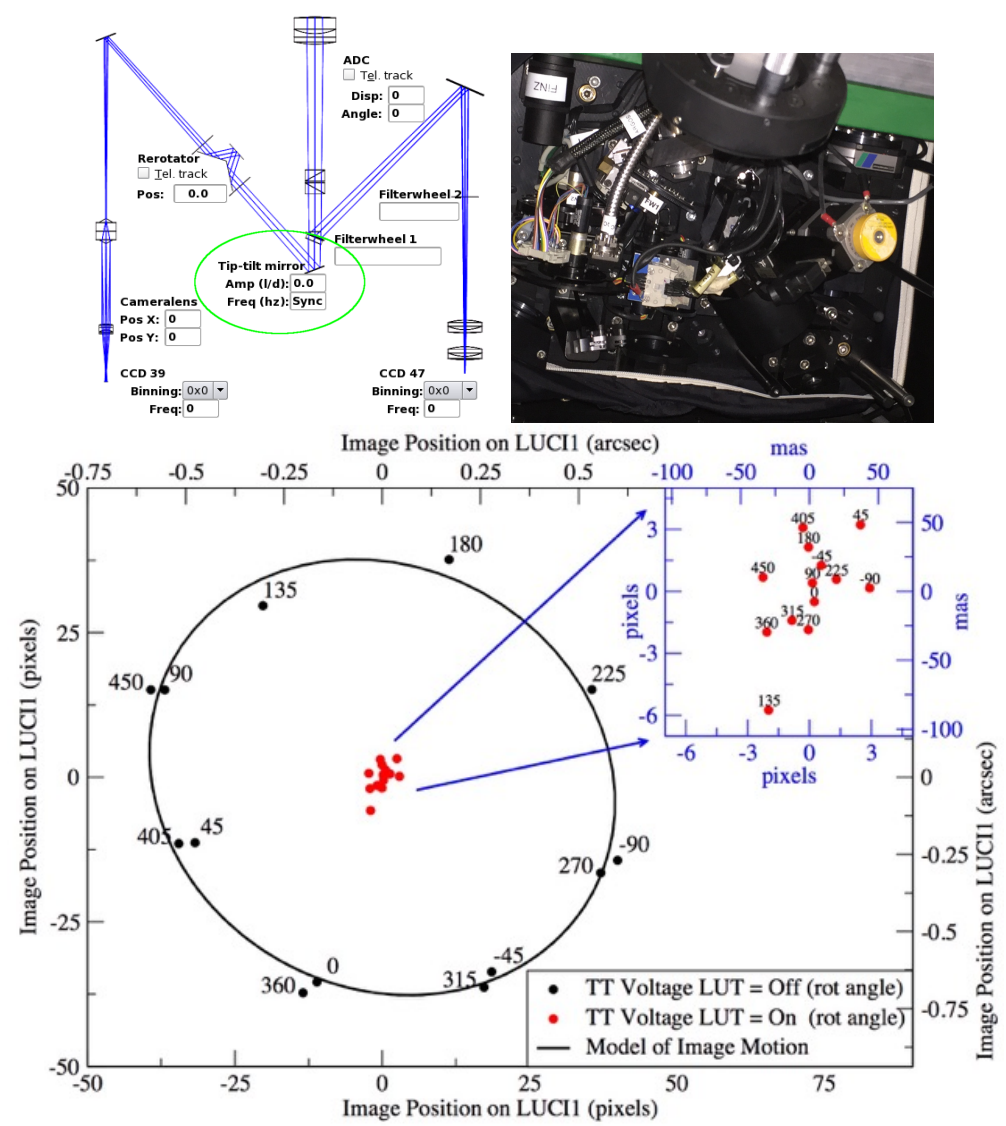

Figure 5. Top Left: The TT mirror that is used to apply the TT LUT. Top Right: An actual photograph of the same area of the FLAO system. Bottom: The image motion before (black) and after (red) the TT LUT is applied.

\section{CONCLUSION}

Work to improve the robustness and ease of use of the FLAO system, at the LBT. This work consists of software improvements that allow the OSA to run the FLAO system with minimal assistance. Other improvements have increased the quality of the observations which can be made with the FLAO system, further increasing the scientific impact of the LBTO.

\section{ACKNOWLEDGMENTS}

The LBT is an international collaboration among institutions in the United States, Italy and Germany. LBT Corporation partners are: The University of Arizona on behalf of the Arizona Board of Regents; Istituto Nazionale di Astrofisica, Italy; LBT Beteiligungsgesellschaft, Germany, representing the Max-Planck Society, The Leibniz Institute for Astrophysics Potsdam, and Heidelberg University; The Ohio State University, and The Research Corporation, on behalf of The University of Notre Dame, University of Minnesota and University of Virginia.

\section{REFERENCES}

[1] Esposito, S., Riccardi, A., Fini, L., Puglisi, A. T., Pinna, E., Xompero, M., Briguglio, R., Quirós-Pacheco, F., Stefanini, P., Guerra, J. C., Busoni, L., Tozzi, A., Pieralli, F., Agapito, G., Brusa-Zappellini, G., Demers, R., Brynnel, J., Arcidiacono, C., and Salinari, P., "First light AO (FLAO) system for LBT: final integration, acceptance test in Europe, and preliminary on-sky commissioning results," in [Adaptive Optics Systems II], 7736, 773609 (July 2010). 
[2] Hill, J. M. and Salinari, P., "Large Binocular Telescope Project," in [Advanced Technology Optical/IR Telescopes VI], Stepp, L. M., ed., 3352, 23-33 (Aug. 1998).

[3] Hill, J. M., Green, R. F., Ashby, D. S., Brynnel, J. G., Cushing, N. J., Little, J. K., Slagle, J. H., and Wagner, R. M., "The Large Binocular Telescope," in [Ground-based and Airborne Telescopes IV], 8444, 84441A (Sept. 2012).

[4] Riccardi, A., Brusa, G., Salinari, P., Gallieni, D., Biasi, R., Andrighettoni, M., and Martin, H. M., "Adaptive secondary mirrors for the Large Binocular Telescope," in [Adaptive Optical System Technologies II], Wizinowich, P. L. and Bonaccini, D., eds., 4839, 721-732 (Feb. 2003).

[5] Christou, J. C., Brusa, G., Guerra, J. C., Lefebvre, M., Miller, D., Rahmer, G., Sosa, R., and Wagner, M., "Living with adaptive secondary mirrors 365/7/24," in [Adaptive Optics Systems IV], 9148, 91480F (Aug. 2014).

[6] Riccardi, A., Bindi, N., Ragazzoni, R., Esposito, S., and Stefanini, P., "Laboratory characterization of a Foucault-like wavefront sensor for adaptive optics," in [Adaptive Optical System Technologies], Bonaccini, D. and Tyson, R. K., eds., 3353, 941-951 (Sept. 1998).

[7] Ragazzoni, R., Ghedina, A., Baruffolo, A., Marchetti, E., Farinato, J., Niero, T., Crimi, G., and Ghigo, M., "Testing the pyramid wavefront sensor on the sky," in [Adaptive Optical Systems Technology], Wizinowich, P. L., ed., 4007, 423-430 (July 2000).

[8] Mandel, H., Appenzeller, I., Bomans, D., Eisenhauer, F., Grimm, B., Herbst, T. M., Hofmann, R., Lehmitz, M., Lemke, R., Lehnert, M., Lenzen, R., Luks, T., Mohr, R., Seifert, W., Thatte, N. A., Weiser, P., and Xu, W., "LUCIFER: a NIR spectrograph and imager for the LBT," in [Optical and IR Telescope Instrumentation and Detectors], Iye, M. and Moorwood, A. F., eds., 4008, 767-777 (Aug. 2000).

[9] Bloemhof, E. E. and Dekany, R. G., "Metrology for the adaptive optics system at the Palomar 200-in. telescope," in [Adaptive Optical System Technologies], Bonaccini, D. and Tyson, R. K., eds., 3353, 638-648 (Sept. 1998).

[10] Noll, R. J., "Zernike polynomials and atmospheric turbulence," Journal of the Optical Society of America (1917-1983) 66, 207-211 (Mar. 1976).

[11] Born, M. and Wolf, E., [Principles of Optics], Cambridge University Press, Cambridge, UK, seventh ed. (1999). 